Revista de Psicología Vol. 39 (2), 2021 (e-ISSN 2223-3733)

\title{
Cumplimiento de medidas de reparación y construcción de cultura de paz en Ecuador y Chile
}

\author{
Carlos Reyes-Valenzuela ${ }^{1}$, Joselyn Bustillos Caranqui ${ }^{2}$; Andrés Aguirre \\ Jaramillo ${ }^{3}$; Rodrigo Moreta-Herrera ${ }^{4}$; Andrés Durán Pereira ${ }^{5}$; Katherine \\ Maturana Iturriaga ${ }^{6}$ y Loreto Villagrán Valenzuela ${ }^{7}$ \\ Universidad Andina Simón Bolivar - Ecuador, Universidad Politécnica \\ Salesiana de Ecuador, Universidad Central del Ecuador-Ecuador, Pontificia \\ Universidad Católica del Ecuador-Ecuador, Universidad Academia de \\ Humanismo Cristiano-Chile, Universidad de Concepción-Chile
}

El objetivo de este trabajo consiste en identificar cómo los impactos de los Informes de Comisiones de Verdad en Chile y Ecuador han promovido el cumplimiento de medidas de reparación integral y su relación con la construcción de la Cultura de Paz (CP). Se realizaron dos estudios: el primero, cuyo objetivo fue diseñar y validar el contenido un instrumento para medir la percepción de cultura de paz. El segundo, busca evaluar el conocimiento de medidas de reparación integral como variable interviniente entre las actitudes al trabajo de

1 Doctor en Ciencias del Comportamiendo y de la Salud. Profesor-investigador en la Universidad Andina Simón Bolívar, Ecuador. Dirección postal: Madrid E12-208 barrio La Floresta, Quito, Ecuador. Contacto: carlos.reyes@uasb.edu.ec. https://orcid.org/0000-0001-8911-700X

2 Licenciada en Psicología. Asistente de investigación en Universidad Politécnica Salesiana, Ecuador. Dirección postal: Pedro de Alvarado n63-38, Quito EC170103, Quito, Ecuador. Contacto: arleth.jb03@gmail.com. https://orcid.org/0000-0003-4299-7964

3 Magíster en Derechos Humanos y Democratización. Profesor en la Universidad Central del Ecuador, Ecuador. Dirección postal: Coruña N13 y N14 y Alberto Mena, Quito, Ecuador. Contacto: andresaguirrejaramillo21@gmail.com

4 Magíster en Psicología. Profesor-investigador en la Pontificia Universidad Católica del Ecuador. Dirección postal: Av. Manuela Sáenz s/n, sector el Tropezón, Ambato, Ecuador. Contacto: rmoreta@pucesa.edu.ec. https://orcid.org/0000-0003-0134-5927

5 Magíster en Psicología Comunitaria. Jefe de Carrera de la Escuela de Psicología en la Universidad Academia de Humanismo Cristiano, Chile. Contacto: aduranp@docentes.academia.cl

6 Magíster en Praxis Comunitaria y Pensamiento Sociopolítico. Coordinadora del Programa Habilidades para la Vida en la Universidad Academia de Humanismo Cristiano, Chile. Contacto: katherine.maturana@gmail.com. https://orcid.org/0000-0001-9844-4707

7 Doctora en Metodología y Ciencias del Comportamiento y de la Salud. Profesora en la Universidad de Concepción, Chile. Dirección postal: Janequeo 421 depto. 85, Concepción, Chile. Contacto: lorevillagran@udec.cl 
las Comisiones y las funciones estatales de CP. Se discuten las implicancias de la falta de cumplimiento de medidas de reparación integral y su relación con la construcción de CP en sociedades posconflictos.

Palabras clave: Posconflicto, Comisiones de Verdad, reparación integral, cultura de paz, Ecuador y Chile

\section{Compliance with measures to repair and build peace culture in Ecuador and Chile}

The objective of this work is to identify how the impacts of the Truth Commissions Reports in Chile and Ecuador have promoted compliance with comprehensive reparation measures and their relationship with the construction of the Culture of Peace (CP). Two studies were carried out: the first one, whose objective was to design and validate the content, an instrument to measure the perception of a culture of peace. The second one seeks to evaluate the knowledge of integral reparation measures as an intervening variable between the attitudes towards the work of the Commissions and the state functions of the CP. The implications of the lack of compliance with comprehensive reparation measures and their relationship with the construction of CP in post-conflict societies are discussed.

Keywords: Post-conflict; Truth Commissions; repair; culture of peace; Ecuador and Chile

\section{Cumprimento das medidas de reparo e construçáo de uma cultura de paz no Equador e Chile}

O objetivo deste trabalho é identificar como os impactos dos Relatórios da Comissão da Verdade no Chile e no Equador promoveram o cumprimento de medidas integrais de reparação e sua relação com a construçáo da Cultura de Paz (CP). Foram realizados dois estudos: o primeiro, cujo objetivo era projetar e validar o conteúdo, um instrumento para medir a percepção de uma cultura de paz. A segunda busca avaliar o conhecimento das medidas de reparação integral como uma variável interveniente entre as atitudes em relação ao trabalho das Comissóes e as funçóes estatais do CP. São discutidas as implicaçóes da falta de cumprimento de medidas abrangentes de reparação e sua relação com a construção da CP nas sociedades pós-conflito.

Palavras-chave: Pós-conflito; Comissóes da Verdade; reparo abrangente; cultura da paz; Equador e Chile

\section{Respect des mesures de réparation et de construction d'une culture de la paix en Equa- teur et au Chili}

L'objectif de ce travail est d'identifier comment les impacts des rapports des commissions de vérité au Chili et en Équateur ont favorisé le respect des mesures de réparation globales et leur relation avec la construction de la culture de la paix (CP). Deux études ont été menées: la première, dont l'objectif était de concevoir et valider le contenu, un instrument pour mesurer la perception d'une culture de la paix. Le second vise à évaluer la connaissance des mesures de réparation intégrale comme variable intermédiaire entre les attitudes à l'égard du travail des commissions et les fonctions étatiques du CP. Les implications du non-respect des mesures de réparation globales et leur relation avec la construction de CP dans les sociétés sortant d'un conflit sont discutées.

Mots-clés: post-conflit; Commissions vérité; réparation complète; culture de paix; Equateur et Chili 
Los estudios sobre los procesos destinados a abordar las graves violaciones de derechos humanos y delitos de lesa humanidad tras periodos de represión y violencia estatal han recibido atención política y académica en América Latina. Las interpretaciones a partir de estos estudios se han centrado en aspectos tales como el reconocimiento de los Estados frente a las violaciones a los derechos humanos, la implementación de mecanismos de reparación integral a las víctimas, las acciones de judicialización de los agentes involucrados o las posibilidades de la construcción de una memoria histórica, entre otros. En el presente trabajo, se aborda una perspectiva que busca complementar los anteriores estudios en justicia transicional, al plantear un análisis de las violaciones de derechos humanos en relación al cumplimiento de los mecanismos de reparación y la construcción de cultura de paz en sociedades latinoamericanas posconflictos.

\section{La justicia transicional y mecanismos de reparación en Amé- rica Latina}

De acuerdo con el International Center for Transitional Justice (ITCJ, 2009) América Latina ha sido un continente pionero en abordar el terrorismo de Estado cuya data viene desde la segunda mitad del siglo XX, con la implementación de la Doctrina de Seguridad Nacional (Feierstein, 2009). En este marco, se plantea el concepto de guerra interna que prevé la destrucción de fuerzas insurgentes y la neutralización de aparatos políticos opositores, impulsados por los Estados con apoyo de las élites económicas y políticas. Si bien el terrorismo de Estado ocurre durante regímenes dictatoriales en numerosos países de la región, ocurre en menor medida durante gobiernos democráticos y, en otros casos, las vulneraciones ocurren en el contexto de conflictos armados internos.

Al hacer una revisión histórica de estos procesos, se observa que desde mediados de los años ochenta, diversos gobiernos de transición en 
América Central y del Sur fueron sustituyendo tales contextos de represión política por modelos de gobernanza democráticos. Esto implica que los Estados consideren dos estrategias para abordar las violaciones de derechos humanos y crímenes de lesa humanidad y promover la consolidación de la democracia: primero, el reconocimiento de las violaciones de derechos humanos, con lo cual plantea una ruptura simbólica con el antiguo régimen, demostrando un compromiso moral y político acorde a los valores democráticos y la esperada reconciliación. Segundo, los Estados adoptan medidas de conciliación y amnistía, sin una investigación de los hechos con el objetivo de evitar nuevos conflictos (Rodrigues, 2017). Bajo este panorama, surgen acciones de "justicia transicional", entendida como un conjunto de mecanismos mediante los cuales se pretende atender las violaciones a los derechos humanos en un contexto de transición a gobiernos democráticos, o en periodos posteriores a conflictos armados (Beristain, Páez, Rimé, \& Kanyangara, 2010). La justicia transicional recoge principios legales, mecanismos y enfoques utilizados para afrontar la violencia del pasado, los cuales se identifican en tres categorías: "mecanismos de rendición de cuentas" que incluyen Comisiones de la Verdad; "mecanismos de justicia restaurativa" orientados al derecho a la reparación de las víctimas y "mecanismos de seguridad y paz", que incorporan reformas normativas e institucionales (Olsen, Payne, \& Reiter, 2016).

En relación a los "mecanismos de rendición de cuentas" implementados en América Latina, se puede revisar en Argentina la Comisión Nacional de Desaparecidos (Conadep), que elabora el Informe "Nunca Más". Sin embargo, el proceso se obstaculiza con la promulgación de la Ley de Obediencia Debida y Punto Final, con las que se indulta a los responsables, situación que intenta modificarse a partir de la década del 2000, con el avance en procesos de judicialización de los victimarios y el establecimiento de programas de reparaciones a las víctimas por vía administrativa. En Chile, se crean dos Comisiones de la Verdad, la Comisión Nacional de Verdad y Reconciliación (CNVR, Comisión Rettig, 1990-1991) y la Comisión Nacional sobre Prisión Política y Tortura (CNPPT, Comisión Valech, 2003-2004) que permiten docu- 
mentar las graves violaciones a los derechos humanos e iniciar procesos de judicialización. En Guatemala y El Salvador se crean Comisiones de la Verdad debido a los conflictos armados internos, cuyas recomendaciones no tuvieron mayor impacto social y fueron limitadas por leyes de amnistía. En la región Andina, se destacan las Comisiones de la Verdad de Perú y Ecuador, caracterizadas por la formulación autoconsciente de políticas por parte de los Estados (Olsen et al., 2016) que a diferencia de anteriores Comisiones, incorporan un análisis de género.

Las Comisiones de la Verdad han sido abordadas por diversos estudios de procesos de justicia transicional que analizan su aporte social en la prevención de los ciclos de venganza, evitar los crímenes de guerra o prevenir el resurgimiento de procesos de violencia colectiva (Cárdenas, Páez, Arnoso, \& Rimé, 2013). No obstante, una de las principales limitaciones se centra en la implementación de "mecanismos de justicia restaurativa" orientados al derecho a la reparación de las víctimas (Resolución sobre impunidad, N²005/81 de la Comisión de Derechos Humanos de Naciones Unidas y Resolución N 60/147 de la Asamblea General de las Naciones Unidas) y en los "mecanismos de seguridad y paz" que desafían al diseño de reformas normativas e institucionales para abordar las violaciones y promover la no repetición de los hechos. Si bien estos mecanismos se constituyen en procesos de relevancia histórica para los países de la región, para las víctimas representan acciones ambivalentes, en las que se generan expectativas de reparación, pero a la vez, se produce un impacto asociado a la entrega de sus testimonios (Beristain et al., 2010). Esto ocurre por la falta de reconocimiento de los Estados y de la sociedad civil del impacto psicosocial de las violaciones de derechos humanos (Beristain, 2007) que deriva en medidas de escaso impacto a la hora de investigar, determinar responsables e implementar mecanismos de reparación integral. Como consecuencia, tal concepción social y política ha provocado que no haya el suficiente impulso social para implementar las recomendaciones de los informes. De allí que el impacto de los procesos de justicia transicional no solo se asocie a la creación de Comisiones de Verdad o la difusión de sus informes (Mathías et al., 2020) sino al grado de conoci- 
miento de las recomendaciones planteadas (Brahm, 2007). De acuerdo con la Comisión Interamericana de Derechos Humanos (2014) la falta de implementación de las recomendaciones de las Comisiones de la Verdad en América Latina tiene un profundo impacto negativo en la reconstrucción de la confianza ciudadana y profundiza las secuelas de las violaciones de derechos humanos cometidas en el pasado.

En el caso de los procesos de reparación en Chile y Ecuador, objetivo de este trabajo, a partir de los Informes de las Comisiones de Verdad se pone en evidencia que el camino de la reparación resulta un tránsito largo para las víctimas y que los Estados suelen aplazar las acciones que involucran la judicialización de los responsables. En el caso de Chile, en el primer informe realizado por la Comisión Nacional de Verdad y Reconciliación en 1990, se posibilita el reconocimiento oficial de la violencia y la reprobación moral del pasado, mediante disculpas institucionales. Asimismo, a partir de las recomendaciones, se crea la Corporación Nacional para la Reparación y Reconciliación, se instaura un plan de compensaciones conformada por pensiones a familiares de las víctimas, becas de estudios, programas de salud mental y física, la edificación de monumentos en los lugares que funcionaban como centros de detención y tortura y, lentamente, se promueven procesos contra agentes de la dictadura (Arnoso, Cárdenas, \& Páez, 2015; Rodrígues, 2017). Posteriormente, con la referida Comisión Valech, se presenta un informe sobre la situación de tortura y en la ley $\mathrm{N}^{\circ}$ 19.992 se establecen medidas de reparación para las víctimas calificadas, las cuales continúan la línea de reparaciones establecida en el primer Informe. De acuerdo a informes de exámenes periódicos universales al país (A/HRC/WG.6/5/CHL/1, 2009; 2019) se ha avanzado en medidas de compensación económica a las víctimas, en el diseño de lugares de memoria, en la creación de un Instituto Nacional de Derechos Humanos y en el seguimiento del gobierno respecto a los procesos pendientes judiciales. Sin embargo, aún no se identifica con exactitud el destino final de personas desaparecidas y, en el último tiempo, se han rebajado penas a algunos responsables de violaciones de derechos 
humanos o han quedado en libertad, bajo una estrategia discursiva estatal que fomenta el perdón y olvido.

En el caso de Ecuador, se formularon 155 recomendaciones clasificadas en medidas de justicia, satisfacción, restitución, rehabilitación, indemnización y garantías de no repetición, se realizaron disculpas y en el 2013, se promulga una Ley de Víctimas. Por otro lado, de acuerdo al último examen periódico universal a Ecuador (2017) se destacan la creación de la Dirección de Reparación a víctimas de graves violaciones a los derechos humanos y se realizan los primeros acuerdos indemnizatorios. No obstante, a la fecha, la principal instancia judicial (Fiscalía General del Estado) no ha logrado investigar el diez por ciento de los casos documentados dificultando el acceso a la justicia que demandan las víctimas. Se evidencia una falta de compromiso y comprensión del Estado sobre las implicaciones al derecho a la reparación integral, por cuanto las instituciones públicas no realizan un análisis de si las medidas resultan justas, apropiadas o efectivas, sino que su preocupación radica únicamente en su legalidad. Las escasas medidas de reparación se canalizan a través de servicios públicos ordinarios lo que conlleva al condicionamiento de las víctimas en aceptar medidas básicas sin mayor desarrollo y que no responden a la proporcionalidad de sus necesidades.

Tomados en conjunto, se pone de manifiesto que las sociedades que transitan hacia la paz requieren de "un conjunto de cambios estructurales o de forma en el funcionamiento del Estado que implican, la mayoría de las veces, a los poderes ejecutivo, judicial o legislativo" (Beristain, 2007, p. 373) los cuales no necesariamente están orientados a los sentidos de reparación que buscan las víctimas. Por el contrario, ambos Estados han configurado un sentido de reparación que está más relacionado a los intereses surgidos en las coyunturas políticas y sociales, con una débil o nula participación de las víctimas, generando escasas acciones para el avance de la memoria histórica de los hechos del pasado (Winn, 2013) y planteando inadecuadamente un imaginario de recuerdo y olvido (Pipper, 2005). Frente a esto último, resulta fundamental centrarse en las garantías de no repetición, componente 
de la reparación integral, que no solo se enfoca en las necesidades de las víctimas, sino que pretende asegurar una repercusión social que impida la repetición de las vulneraciones y fomente el respeto y promoción de los derechos humanos (Beristain, 2009; García Ramírez, 2005). Así, la consideración y cumplimiento de los Estados de las garantías de no repetición promoverán mejores condiciones para la construcción de cultura de paz.

\section{Reparación integral y construcción de cultura de paz en sociedades posconflicto}

La noción de Cultura de Paz (CP) está frecuentemente asociada a develar las dinámicas de violencia y al cese de conflictos. No obstante, puede ser mejor comprendida como los esfuerzos y acciones de una cultura para orientar e implementar un mundo más pacífico (Muñoz \& Molina, 2010). La Declaración Universal de Derechos Humanos (1948) configura una declaración de intenciones por construir un modelo de paz que posibilite las condiciones para hablar y pensar en CP (Muñoz, 2007). Las posteriores consideraciones de Unesco (1998) y la Declaración y Programa de Acción sobre CP (A/RES/53/243, 6 de octubre de 1999) definen CP como:

[...] valores, actitudes y conductas que plasman y suscitan a la vez interacciones e intercambios sociales basados en principios de libertad, justicia y democracia, todos los derechos humanos, la tolerancia y la solidaridad, que rechazan la violencia y procuran prevenir los conflictos tratando de atacar sus causas para solucionar los problemas mediante el diálogo y la negociación y que garantizan el pleno ejercicio de todos los derechos y proporcionan los medios para participar plenamente en el proceso de desarrollo de su sociedad (Naciones Unidas, 1998, p. 1).

Sin embargo, esta definición de CP ha sido identificada como ambigua y difusa, que se plantea en contraposición a la presencia de conflictos en el mundo. De acuerdo a Muñoz y Molina (2010, p. 45) la posibilidad de "gestionar una Cultura de Paz no es una tarea fácil, sino 
que se requieren esfuerzos individuales e institucionales, académicos y científicos, solidarios y cooperativos". Por tanto, su gestión implicaría no solo a los Estados, sino también a las acciones de sociedad civil. De acuerdo a estos autores, la CP debería ser flexible acorde a los cambios que estén sucediendo, a las capacidades que pueden desarrollarse mediante interacciones recíprocas y plantear la existencia de objetivos, valores compartidos, la conciencia de grupo e identificación social.

Por otro lado, el cumplimiento de los Estados respecto al mecanismo de reparación integral resulta indispensable para generar un marco de respeto y garantía de los derechos humanos que posibilite la construcción de cultura de paz (Reyes, Bustillos, \& Aguirre, 2019). Esto plantea tres interrogantes: primero, en la medida que las medidas de reparación integral no cuenten con participación de las víctimas y de la sociedad civil, la construcción de CP será entendida como una tarea exclusiva de los Estados. Segundo, el cumplimiento de la dimensión de judicialización se ha visto expresada como una voluntad política de los Estados, dado que, en muchos casos, los responsables de violaciones de derechos humanos permanecen como miembros activos. Como consecuencia, la construcción de $\mathrm{CP}$ en sociedades posconflicto resulta aún más compleja y conflictiva de lo que ha sido identificada (Muñoz \& Molina, 2010) sentando las bases sobre los criterios de selectividad que los Estados han operado frente a la judicialización. En tercer término, el incumplimiento y la falta de seguimiento de los Estados al carácter integral de las reparaciones relega la visibilidad de las dimensiones de restitución, satisfacción y, fundamentalmente, de las garantías de no repetición.

Respecto de esta última dimensión, las Directrices de van Boven (1993) identifican las garantías de no repetición como aspecto clave para considerar las alternativas en la prevención del surgimiento de nuevos conflictos: en este punto, resultan de interés las directrices que destacan la promoción de la educación en el conocimiento y respeto de los derechos humanos, la promoción de mecanismos para el abordaje de conflictos sociales y la revisión de leyes que posibiliten nuevos escenarios de violaciones de derechos humanos. Estos criterios de garantías 
de no repetición conforman una base para la construcción de CP en sociedades posconflictos, que se sintetizarían en cuatro aspectos: primero, en el reconocimiento de las víctimas y en el acceso y efectivo cumplimiento a medidas de reparación con carácter integral; segundo, a superar la retórica de la reconciliación entendida como perdón interpersonal y grupal y centrarse en la construcción de CP (Cárdenas et al., 2013); tercero, en adoptar diversos mecanismos que corrijan las desigualdades estructurales que generan exclusión, marginación y ausencia del Estado, dinámicas que se observan en diversas realidades en la región (Dajer, 2017); cuarto, en la adopción de una mirada crítica sobre los aspectos que promovieron los hechos de violencia y el análisis sobre los valores que permitan el desarrollo de una convivencia democrática pacífica (Lira, 2010).

Aunque los actuales procesos políticos, sociales y económicos de Ecuador y Chile resultan distintivos, no obstante, las actuales condiciones de corrupción generalizada, en un contexto de permanente vulnerabilidad económica, el creciente clima de protesta y de manifestaciones sobre políticas estatales, la judicialización de líderes y defensores de derechos humanos, la adopción de enfoques securitistas frente a población en movilidad humana y la desprotección que presentan grupos tales como niños, nińas, adolescentes y personas privadas de libertad (Human Right Watch, Informe 2018 Ecuador; Instituto Nacional de Derechos Humanos, Informe Anual situación de los derechos humanos en Chile, 2018 y 2019) están desafiando el desarrollo de políticas y mecanismos institucionales acordes a un marco de respeto y garantías de derechos humanos (Reyes et al., 2019). En este contexto, se reconocen enormes dificultades para abordar la violencia institucional presente en cada país, que originan una alta desconfianza hacia las autoridades políticas y condiciones de vida cada vez más difíciles de asumir.

En este contexto, el estudio presenta como objetivo identificar cómo los impactos de los Informes de Comisiones de Verdad en Chile y Ecuador han promovido el cumplimiento de medidas de reparación integral y, bajo estas condiciones, cómo se relaciona con 
la construcción de CP. La hipótesis central es que a partir del trabajo de las Comisiones de Verdad en América Latina, se plantea que las acciones de cumplimiento de las medidas de reparación integral desde los Estados favorecerán las funciones estatales de CP. A partir de esto, se evalúa las relaciones con la actitud hacia el trabajo de las Comisiones y las acciones de reparación integral (en Chile) o el conocimiento de las recomendaciones de reparación integral (en Ecuador). A partir de este objetivo, se plantean dos estudios: el primero orientado al diseño y validación de una encuesta que identifique dimensiones psicosociales de cultura de paz (EDPCP) y, en un segundo estudio, se aborde la relación entre los impactos de los informes, las medidas de reparación integral y la construcción de CP.

\section{ESTUDIO 1a \\ Diseńo de ítems, validez de constructo de la Escala Dimensiones psicosociales de Cultura de Paz (EDPCP)}

\section{Método}

\section{Procedimiento}

Construcción de los items. La construcción de ítems de la EDPCP fue realizado a partir de una definición conceptual de dimensiones psicosociales de cultura de paz. En la construcción, se definió operativamente cultura de paz como: "aquella en que se valoran los derechos humanos, todo ciudadano tiene un trato digno, se acepta el conflicto y las diferencias, pero no se acepta la violencia y represión como método de resolverlos y de acción política”. Esta definición se asoció a políticas, iniciativas y acciones realizadas que contribuyan en la construcción de cultura de paz. En este punto, se diferenció la acción de los Estados orientado a generar política de cultura de paz de las realizadas por sociedad civil, entendidas como iniciativas y propuestas de paz no formal ni institucionalizada. Posteriormente, se realizó una revisión de 
los 30 ítems construidos que cumplían algunos de los requisitos sugeridos para la construcción de escalas (Abad, Olea, Ponsoda, \& Garcia, 2011): a) se cuidó en su redacción el uso de los tiempos verbales b) se atendió que el contenido de los ítems fuera teóricamente relevante en torno a la noción de cultura de paz y la dimensión que representaban; d) se utilizó un lenguaje claro e inteligible teniendo en cuenta las muestras a las que iban a ser aplicados (por ejemplo, no se usan siglas tales como LGBTI). Para evaluar el contenido de los 30 ítems construidos, se realizó un muestreo intencional en un grupo de tres expertos en derechos humanos que trabajan en Ecuador, Chile y España, los cuales seleccionaron los ítems e hicieron comentarios respecto a las instrucciones, aspectos omitidos, redacción y adecuación del formato de respuesta, los cuales fueron posteriormente considerados a la hora de la redacción definitiva de los ítems.

\section{Medición}

El instrumento requería una validación de las distintas dimensiones psicosociales de la cultura de paz a través de criterios de jueces. Para este fin, se establecieron instrucciones para abordar las dimensiones seleccionadas, en el cual la tarea de los jueces consistía en responder a la siguiente propuesta: en primer lugar, la pertinencia del ítem respecto al constructo de dimensiones psicosociales de cultura de paz (IP) el cual presentaba un formato de respuesta entre 1 (ítem poco o nada pertinente con el constructo propuesto) y 10 (ítem muy pertinente con el constructo propuesto).

Segundo, para evaluar el acuerdo de los jueces respecto a los 30 ítems propuestos, se optó por el índice de congruencia (IC) de acuerdo ítem-objetivo de Rovinelli y Humbleton (1977) en el cual se presentaba un formato de respuesta entre -1 (ítem nada congruente con la dimensión propuesta), 0 (ítem poco congruente con la dimensión propuesta) y 1 (ítem muy congruente con la dimensión propuesta). Este indicador es uno de los más utilizados y recomendados para evaluar el grado en que los ítems son representativos de un dominio a evaluar 
(Abad et al., 2011; Villagrán, 2016), lo que permitió seleccionar una muestra representativa de ítems a partir de puntos de corte.

\section{Análisis de datos}

Para evaluar la pertinencia o la relevancia (IP) se obtuvo para cada ítem una valoración por parte de cada uno de los tres jueces. Para el IP se calculó la puntuación media de cada ítem, sumando la puntuación que le asignó cada juez al ítem en cuanto a su pertinencia o relevancia con el constructo de CP, dividido por el número total de jueces (3). Para calcular el IC se estableció la siguiente variación respecto del procedimiento original: cada ítem fue evaluado por los tres jueces en el cual respondieron a la congruencia que presentaba con el constructo de dimensiones psicosociales propuestas de cultura de paz. Con los puntajes asignados por todos los jueces para cada ítem se calculó el IC.

\section{Resultados y Discusión}

Para cada ítem se calculó el IP y luego se procedió a seleccionar los que serían incorporados en la EDPCP, para su aplicación. A tal efecto, se establecieron dos criterios: Un punto de corte de aquellos ítems que tuvieran un IP $\geq 7.0$ y de cada IC $\geq 0.6$. Se obtuvo que 25 ítems cumplían con estos criterios, de los cuales cuatro se incluyeron en la dimensión "funciones estatales de cultura de paz"; seis ítems en la dimensión "factores que afectan la cultura de paz"; cinco ítems en la dimensión "iniciativas de la sociedad civil que promueven la cultura de paz; y 10 ítems en la dimensión "personas y grupos prioritarios en las políticas de cultura de paz del Estado". Luego de obtener tales ítems, resultó necesario explorar cómo se agrupan en las dimensiones propuestas, examinar la estructura factorial y la consistencia interna de la EDPCP que se indagó en el estudio $1 \mathrm{~b}$. 


\section{ESTUDIO 1b \\ Validez factorial y consistencia interna de la EDPCP}

\section{Método}

\section{Participantes}

Participaron voluntariamente 1011 adultos, distribuidos en 544 que viven en Ecuador y 467 en Chile. La muestra estuvo conformada por 509 mujeres (50.3\%) y 502 hombres (49.7\%), con una media de edad de 38.6 años $(\mathrm{DE}=17.7)$ con edades que varían entre 15 y 89 años. El muestreo fue por cuotas en sexo y edad, lo que posibilitó una mayor flexibilidad y representatividad (Rodríguez-Osuna, 1991) para lo cual se utilizaron los datos censales de Ecuador $(2010)^{18}$ y Chile $(2017)^{9}$. Los participantes no recibieron compensación económica por sus respuestas.

\section{Procedimiento}

Se establecieron dos métodos de recolección de la información: en primer lugar, se diseñó una encuesta escrita que fue contestada por el 58\% del total de la muestra, en tanto, una segunda versión online fue respondida por el $42 \%$. En ambos casos, la encuesta fue aplicada entre los meses de abril a septiembre de 2018 en las ciudades de Quito y Santiago.

8 En Ecuador, la cuota por sexo, es de 50\% para hombres y $50 \%$ para mujeres. En el caso de la edad, se establecieron tres categorías: 15-24 ańos, conformado por un 25\%, 25-55 años, por un $46 \%$ y más de 56 ańos, compuesto por un $29 \%$. El estudio se sitúa en el Distrito Metropolitano de Quito, por lo que tal estrategia de muestreo resultó únicamente representativa para tal Distrito Metropolitano y no para el país.

9 En Chile, la cuota por sexo, es de 50\% para hombres y $50 \%$ para mujeres. En el caso de la edad de igual modo que se utilizó en Ecuador, se definieron tres categorías: 15-24 años, conformado por un $21 \%, 25-55$ ańos, por un $48 \%$ y más de 56 años, compuesto por un $31 \%$. El estudio se sitúa en la Región Metropolitana de Santiago, por lo que, de igual modo que en la evaluación en Ecuador, el muestreo fue representativo para tal Región y no el país. 


\section{Análisis de datos}

Para evaluar la estructura factorial de la EDPCP, se utilizó como método de extracción de factores el de factorización del eje principal (Izquierdo, Olea y Abad, 2014) por su eficacia frente a otros métodos. En cuanto a la rotación, se utilizó la rotación oblimin directa (rotación oblicua) asumiendo que los factores están correlacionados (Izquierdo et al., 2014) dado que suele ser común que se observe una vinculación en estudios en ciencias sociales. Se utilizó, además, la prueba de adecuación muestral de Kaiser Meyer-Olkin (KMO) para verificar la idoneidad de los datos para realizar el Análisis Factorial Exploratorio (AFE). Se adoptó el criterio de Kaiser (1974) según el cual valores superiores de $\mathrm{KMO}>0,80$ resultan convenientes y la prueba de esfericidad de Bartlett adoptando $p<0,05$. Además, se consideraron los siguientes criterios para la depuración de la escala: (1) la carga factorial de los ítems en los factores debía ser igual o superior a 0.3 y (2) se realizó un análisis de consistencia interna a través del estadístico Alfa de Cronbach, el cual se planteó superior a 0.7 (Nunnally y Bernstein, 1994). El AFE se realizó con el 25\% de la muestra a través del Programa Factor (Ferrando y Lorenzo-Seva, 2017) y el análisis de consistencia interna con el paquete estadístico SPSS 22 para Windows. El segundo procedimiento correspondió al Análisis Factorial Confirmatorio (AFC) por el método de Máxima Verosimilitud para probar el modelo obtenido del AFE. Para este análisis se consideró el restante $75 \%$ de la muestra. Se probaron distintos modelos de ajuste a través del Chi cuadrado $(\chi 2)$ y el Chi cuadrado normado ( $\chi 2 / \mathrm{Df})$ (Hu \& Bentler, 1999) y los índices de Bondad de Ajuste (GFI), de Ajuste Comparativo (CFI) y Ajuste Normado (NFI), en el que valores superiores a .90 se conformaron como indicadores de un buen ajuste (Hair, Anderson, Tatham \& Black, 2004) y el Error Cuadrático Medio de Aproximación (RMSEA) > .06 (Batista-Fogueta, Manuel, Coenders \& Alonso, 2004; Thompson, 2004). La gestión informática del trabajo se desarrolló con el software $\mathrm{r}$ (R Core Team, 2019). 


\section{Resultados y Discusión}

\section{Análisis normalidad multivariada y multicolinealidad}

Los resultados se muestran en la Tabla 1. En el análisis de normalidad multivariada, los puntajes de la curtosis se encontraron entre -0.772 en el ítem seis y -0.074 en el ítem 25 ; mientras que en el caso de la asimetría estos permanecieron entre -0.416 en el ítem nueve y 0.590 en el ítem 25. Respecto al supuesto, se puede considerar que se cumple, ya que los puntajes de dispersión se encontraron dentro del umbral \pm 1.5 (George \& Mallery, 2003). Por otra parte, en el análisis de multicolinealidad, a través del análisis de correlaciones ítem-test corregidas, estas muestran que son homogéneas con valores extremos entre 0.165 en el ítem 12 y 0.711 en el ítem 17 y que permite identificar la ausencia de redundancias entre los ítems o bajas correlaciones e impide la eliminación de ítems por estos causales. Es decir, no se presenta la multicolinealidad.

\section{Análisis factorial}

Análisis factorial exploratorio. Para el cálculo del análisis factorial exploratorio (AFE) se consideró segmentar a la muestra con un grupo de 250 participantes, considerando el criterio de colocar cinco participantes por cada ítems presente en el cuestionario (Ferrando \& Anguiano-Carrasco, 2010). El AFE con el método de extracción componentes principales y con rotación Oblimin directo muestra la conformación de tres factores que en conjunto explican el 62,8\%. Los resultados se describen en la Tabla 2. 


\section{Tabla 1}

Análisis preliminar de los items y normalidad multivariada

\begin{tabular}{|c|c|c|c|c|c|c|}
\hline Ítems & $M$ & $D E$ & Curtosis & Asimetría & $\boldsymbol{r}\left(_{\text {item-test })}\right.$ & $\alpha$ \\
\hline IT1 & 1.95 & 0.09 & -0.088 & 0.518 & .603 & .939 \\
\hline IT2 & 2.01 & 0.08 & -0.354 & 0.307 & .694 & .939 \\
\hline IT3 & 2.30 & 0.10 & -0.646 & -0.001 & .643 & .938 \\
\hline IT4 & 1.93 & 0.09 & -0.369 & 0.453 & .610 & .938 \\
\hline IT5 & 2.65 & 0.11 & -0.622 & -0.144 & .289 & .941 \\
\hline IT6 & 2.73 & 0.11 & -0.772 & -0.229 & .283 & .941 \\
\hline IT7 & 2.59 & 0.11 & -0.600 & -0.071 & .371 & .940 \\
\hline IT8 & 2.71 & 0.12 & -0.735 & -0.193 & .265 & .941 \\
\hline IT9 & 2.96 & 0.11 & -0.815 & -0.416 & .329 & .941 \\
\hline IT10 & 2.65 & 0.11 & -0.622 & -0.144 & .289 & .941 \\
\hline IT11 & 2.53 & 0.12 & -0.858 & -0.015 & .364 & .941 \\
\hline IT12 & 2.19 & 0.12 & -1.045 & 0.295 & .165 & .942 \\
\hline IT13 & 1.94 & 0.11 & -0.547 & 0.545 & .488 & .939 \\
\hline IT14 & 2.11 & 0.11 & -0.549 & 0.393 & .504 & .939 \\
\hline IT15 & 1.78 & 0.11 & -0.081 & 0.818 & .522 & .939 \\
\hline IT16 & 1.94 & 0.09 & -0.078 & 0.353 & .582 & .939 \\
\hline IT17 & 2.33 & 0.10 & -0.467 & 0.004 & .711 & .938 \\
\hline IT18 & 2.33 & 0.11 & -0.526 & -0.015 & .669 & .938 \\
\hline IT19 & 2.08 & 0.10 & -0.441 & 0.339 & .639 & .938 \\
\hline IT20 & 2.10 & 0.11 & -0.716 & 0.282 & .563 & .939 \\
\hline IT21 & 2.15 & 0.11 & -0.389 & 0.362 & .610 & .938 \\
\hline IT22 & 2.24 & 0.10 & -0.653 & 0.166 & .646 & .938 \\
\hline IT23 & 2.43 & 0.10 & -0.612 & -0.037 & .630 & .938 \\
\hline IT24 & 2.15 & 0.11 & -0.634 & 0.263 & .658 & .938 \\
\hline IT25 & 1.97 & 0.10 & -0.074 & 0.590 & .560 & .939 \\
\hline
\end{tabular}

Nota: $r_{\text {(item-test) }}:$ Correlación item-test corregida 


\section{Tabla 2}

Análisis Factorial Exploratorio de la EDPCP

\begin{tabular}{|c|c|c|c|c|}
\hline Ítems & $\mathbf{h}^{2}$ & \multicolumn{3}{|c|}{ Componente } \\
\hline IT1 & .903 & 0.77 & & \\
\hline IT2 & .941 & 0.96 & & \\
\hline IT3 & .857 & 0.90 & & \\
\hline IT4 & .878 & 0.79 & & \\
\hline IT16 & .940 & 0.74 & & \\
\hline IT17 & 1.00 & 0.74 & & \\
\hline IT18 & .916 & 0.68 & & \\
\hline IT19 & .758 & 0.45 & & \\
\hline IT20 & .899 & 0.74 & & \\
\hline IT21 & .801 & 0.69 & & \\
\hline IT22 & .972 & 0.68 & & \\
\hline IT23 & .923 & 0.63 & & \\
\hline IT24 & .855 & 0.63 & & \\
\hline IT5 & .827 & \multicolumn{3}{|c|}{0.56} \\
\hline IT6 & .871 & \multicolumn{3}{|c|}{0.66} \\
\hline IT7 & .770 & \multicolumn{3}{|c|}{.067} \\
\hline IT8 & .805 & \multicolumn{3}{|c|}{0.66} \\
\hline IT9 & .918 & \multicolumn{3}{|c|}{0.83} \\
\hline IT10 & .725 & \multicolumn{3}{|c|}{0.70} \\
\hline IT11 & .732 & & & 0.64 \\
\hline IT12 & .467 & & & 0.64 \\
\hline IT13 & .784 & & & 0.55 \\
\hline IT14 & 1.00 & & & 0.52 \\
\hline \multirow[t]{2}{*}{ IT15 } & .938 & & & 0.51 \\
\hline & Varianza & 38.5 & 15.3 & 7.5 \\
\hline
\end{tabular}

Nota: $\mathrm{N}=332$ 
Con relación al análisis de consistencia interna, el alfa de Cronbach para la escala completa fue de .91 , en tanto que por cada dimensión los resultados fueron los siguientes: Factor 1, $\alpha=.93$; Factor 2, $\alpha=.87$ y Factor $3, \alpha=.72$. De este modo, la consistencia interna de la escala general como de cada dimensión resulta altamente satisfactoria (Pardo \& San Martín, 2010).

Análisis factorial confirmatorio. Los resultados se muestran en la Tabla 3. De los cuatro modelos probados, se encontró que el mejor ajuste está en el modelo de cuatro factores que previamente fue hipotetizado (el ítem 11 fue retirado por su baja carga factorial), aunque no correspondió al modelo procedente del AFE. Los resultados muestran un ajuste robusto (WLSMV) como adecuado con $\chi 2=443.8 ; \mathrm{p}<.001$; $\mathrm{Df}=224$, así como el estimado Chi Cuadrado Normado $\chi 2 / \mathrm{Df}=2.0$. También en los índices de ajuste GFI= .93; CFI= .92; $\mathrm{SRMR}=.048$; y RMSEA $=.04$ [.035 - .046] según lo estimado por diversos estudios (Abad et al., 2011; Byrne, 2006; Hair et al., 2004) como se observa en la Tabla 4, por lo que se concluye un ajuste aceptable de la EDPCP. A partir de estos componentes, los factores mantienen su identificación conceptual, y son catalogados como sigue: un plano macrosocial conformado por el Factor 1: Funciones estatales en CP y el Factor 2: Aspectos que afectan la CP. Luego, un segundo plano microsocial conformado por el Factor 3: Iniciativas de sociedad civil en relación a CP y el Factor 4: Personas y grupos prioritarios en las políticas de cultura de paz del Estado.

\section{Tabla 3}

Modelos de ajuste de la Escala EPCP con estimación de Minimos Cuadrados Ponderados con Media y Varianza Ajustada (WLSMV)

\begin{tabular}{lccccccl}
\hline Modelo & \multicolumn{1}{c}{$\chi^{2}$} & Df & $\mathrm{X}^{2} / \mathrm{df}$ & CFI & NFI & \multicolumn{1}{l}{ SRMR } & RMSEA \\
\hline Un factor & $2422.4^{* * *}$ & 252 & 9.6 & .36 & .30 & .130 & $.12[.115-.124]$ \\
Tres factores & $719.8^{* * *}$ & 226 & 3.2 & .85 & .83 & .066 & $.06[.055-.065]$ \\
Cuatro factores & $679.1^{* * *}$ & 246 & 2.8 & .87 & .86 & .060 & $.05[.049-.059]$ \\
$\begin{array}{l}\text { (24 ítems) } \\
\text { Cuatro factores }\end{array}$ & $443.8^{* * *}$ & 224 & 2.0 & .93 & .92 & .048 & $.04[.035-.046]$ \\
$(23$ ítems) & & & & & & & \\
\hline
\end{tabular}

Nota: ${ }^{* * *} p<.001 ; \mathrm{n}=604$ 
Los resultados obtenidos mediante el AFE sugieren una estructura de tres factores y el AFC de cuatro factores, este último que coincide con el planteamiento conceptual por lo cual se obtiene una escala que posibilita explorar las dimensiones psicosociales de la cultura de paz, tanto en un plano macro y microsocial. Esto se corrobora en el buen ajuste presentado por la EDPCP, en la alta consistencia interna y validez de la escala. De este modo, se conforma como un instrumento fiable para realizar estudios sobre las dimensiones psicosociales de CP en contextos de la región.

El siguiente estudio retoma la hipótesis de que las medidas de reparación integral puede configurarse como una variable mediadora entre la actitud ante las Comisiones y una de las dimensiones psicosociales de CP: las funciones estatales. Se ha optado por esta última dimensión, puesto que enfatiza en cómo son percibidas las funciones del Estado respecto a CP, en lo referido en cuatro aspectos: la reducción de la violencia, la reducción de la desigualdad, el cuidado de personas y grupos prioritarios y una mejor relación entre el Estado y sociedad civil.

\section{ESTUDIO 2}

La reparación integral como variable interviniente entre las actitudes ante los Informes de Comisiones de Verdad y las funciones estatales de cultura de paz

\section{Método}

\section{Medición}

1. Actitud ante las Comisiones (Gibson, 2004). Evalúa el grado de aprobación de las actividades de las comisiones en las cuatro funciones identificadas: ayuda a las víctimas, ayuda a crear un relato común de las violaciones, ayuda a identificar y judicializar a responsables de las violaciones y ayuda en garantía de no repetición. El rango de respuestas varía entre 1 (nada) a 4 (muchísimo). Presenta una fiabilidad satisfactoria $(\alpha=.87)$. 
2. Conocimiento de las recomendaciones de la CVE. En participantes ecuatorianos, se pregunta por el grado de conocimiento de las recomendaciones que plantea el Informe de la CVE. El rango de respuestas varía entre 1 (nada) a 4 (muchísimo). Presenta una fiabilidad altamente satisfactoria $(\alpha=.91)$.

3. Conocimiento de las medidas de reparación a víctimas de la CNVR y CNPPT. En participantes chilenos, se pregunta por la percepción sobre las medidas de reparación a víctimas que aparecen en los Informes de la CVR y CNPPT. El rango de respuestas varía entre 1 (nada) a 4 (muchísimo). Presenta una fiabilidad altamente satisfactoria $(\alpha=.91)$.

4. Escala de Dimensiones Psicosociales de Cultura de Paz. Constó de 25 preguntas. El rango de respuestas varía entre 1 (nada) a 4 (muchísimo). Presenta una fiabilidad altamente satisfactoria $(\alpha=.91)$.

\section{Procedimiento}

El procedimiento de recolección de información en ambos países se realizó en dos partes: una primera en que los participantes contestaron la encuesta en versión física y una segunda en que se implementó una versión en línea, en las que se controló las cuotas de edad y sexo señaladas con anterioridad. En Ecuador, se invitó a participar voluntariamente utilizando distintas vías de captación: calle, universidades, plazas y algunas instituciones. Luego, cumplido el $70 \%$, se procedió a divulgar el link con la encuesta, principalmente en grupos específicos para lograr la representatividad calculada. Respecto a Chile, los participantes contestaran la versión física de la encuesta, para lo cual, se establecieron contactos con población general en Santiago a través de un trabajo de encuestadores coordinados por un docente de la Universidad Humanismo Cristiano. En el caso de adultos mayores, fueron encuestados en un centro que da atención a necesidades específicas para este grupo. En base a este procedimiento, se obtuvo el $70 \%$ de la muestra total. Posteriormente, se implementó la versión en línea que fue contestada por el restante $30 \%$, mayoritariamente por población joven. En ambas aplicaciones, se consideró una hoja informativa y consentimiento informado para participar en el estudio y se atendió 
el tamańo de la muestra de acuerdo a las variables de sexo y el rango etáreo. La aplicación se realizó entre los meses de abril a septiembre de 2018 en la ciudad de Santiago. Se mantuvo la línea con otras investigaciones en Comisiones de Verdad de incluir un texto informativo sobre el trabajo de la Comisión y de las medidas de reparación, de tal modo que el participante se situara en el tema de investigación.

\section{Análisis de datos}

Con el objetivo de contrastar las hipótesis de mediación, esto es, evaluar en qué medida el conocimiento de las recomendaciones (para el caso de Ecuador) y las percepciones sobre las medidas de reparación integral (en el caso de Chile) desempeñan un rol mediador respecto a la actitud ante el trabajo de las Comisiones de Verdad y las funciones estatales de CP. Se utilizó el macro PROCESS de SPSS (Hayes, 2017). Todos los análisis estadísticos fueron realizados con el programa SPSS 21 y se estableció la significancia estadística en un valor $p<.05$.

\section{Resultados}

\section{Descriptivos}

En la muestra de Ecuador, el $88.4 \%$ de los participantes conoce poco o nada del Informe de la CVE y un $14.5 \%$ ha leído el Informe. En relación a las funciones de las Comisiones de Verdad, un 30\% del total de la muestra refiere que el Informe de la CVE ayudó en el reconocimiento de las víctimas, en la creación de un relato común, en el juzgamiento de responsables y en fomentar garantías de no repetición. Respecto a la información o lectura que presenta de las recomendaciones del Informe, un $14 \%$ de participantes refiere conocerlas. Por último, el $24 \%$ considera que las políticas del Estado han generado una reducción de la violencia en el país; el $27.1 \%$ cree que ha ocurrido una reducción de la desigualdad social; un $42.9 \%$ cree que el Estado ha asumido más políticas de cuidado a grupos de atención prioritaria y el $24.6 \%$ considera que hay una mejor relación entre el Estado y los ciudadanos. 
En el caso de Chile, se consulta en relación a los Informes de CNVR (1991) y CNPPT (2013), igualmente un 30\% de los participantes conoce poco o nada y un $13.5 \%$ presenta un conocimiento de tales Informes. Alrededor del $45 \%$ de los participantes conoce el aporte de ambos Informes en el reconocimiento de las víctimas y creación de un relato común de los hechos, sin embargo, un $28.9 \%$ cree que ayudaron a que los responsables fueran juzgados y un $31 \%$ considera que los Informes han ayudado a garantizar la no repitencia de los hechos. Respecto al grado de conocimiento de la implementación de políticas de reparación integral, el 25.6\% considera que el Estado chileno ha generado políticas de reparación integral, el $28.6 \%$ conoce que se ha cumplido con la compensación económica a las víctimas, un $14.8 \%$ conoce del acceso a apoyo médico y psicológico. Asimismo, un 5.4\% considera que las medidas del Estado han ayudado a cerrar heridas del pasado, el $10.5 \%$ considera que las medidas hacia familiares y víctimas indirectas han sido suficiente y el $19.7 \%$ considera que la sociedad chilena ha propiciado mecanismos de reparación integral. Finalmente, el $17.1 \%$ considera que las políticas del Estado ha generado una reducción de la violencia en el país; el 9.9\% cree que ha ocurrido una reducción de la desigualdad social; el $46 \%$ cree que el Estado ha asumido más políticas de cuidado a grupos de atención prioritaria y el $1.8 \%$ considera que hay una mejor relación entre el Estado y los ciudadanos.

\section{Análisis de mediación}

Se realizaron dos análisis de mediación, uno por país: en Ecuador, si el conocimiento de las recomendaciones que aparecen en el Informe de la Comisión de la Verdad de Ecuador era un factor mediador entre las actitudes ante el trabajo de las Comisiones y las funciones estatales de CP. Los resultados se presentan en la Figura 1. Se encontró que, en primer lugar, la actitud ante el trabajo de las Comisiones se asoció positivamente con la percepción de las medidas de reparación integral $(\mathrm{B}=.18, \mathrm{t}(354)=7.50, \mathrm{p}=.0001)$. Por otro lado, la información de recomendaciones de reparación no se relacionó significativamente con las funciones estatales de $\mathrm{CP}(\mathrm{B}=-.02, \mathrm{t}(354)=-.26, \mathrm{p}=.788)$. 
Por último, la actitud al trabajo de las Comisiones se relacionó positivamente con las funciones estatales de $\mathrm{CP}(\mathrm{B}=.42$, $\mathrm{t}(354)=8.42$, $\mathrm{p}=$ .0001). De este modo, la variable interviniente no explica el efecto de la actitud ante las Comisiones con las funciones estatales de CP.

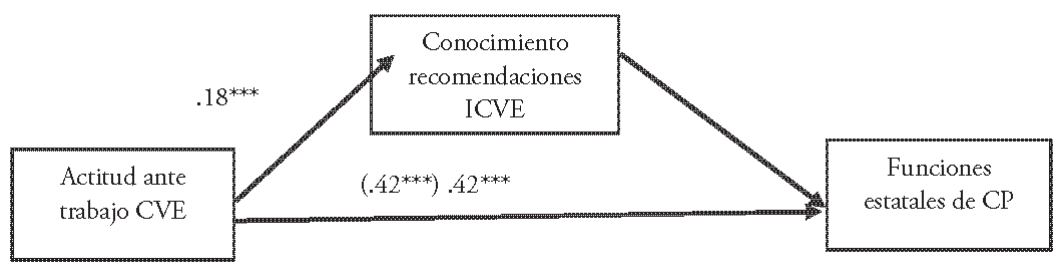

Figura 1. Modelo de mediación parcial entre el conocimiento de las recomendaciones del ICVE, las actitudes del trabajo de la Comisión y las funciones estatales de CP en Ecuador

Nota : CVE = Comisión de la Verdad de Ecuador; ICVE = Informe de la Comisión de la Verdad de Ecuador.

Un segundo análisis de mediación, en Chile, evaluó si la percepción de las medidas de reparación integral realizadas por el Estado era un factor mediador entre las actitudes ante el trabajo de la CNVR y CNPPT y las funciones estatales de CP. Los resultados se presentan en la Figura 2. Se encontró que, en primer lugar, la actitud ante el trabajo de las Comisiones se asoció positivamente con la percepción de las medidas de reparación integral $(\mathrm{B}=.42, \mathrm{t}(463)=8.10, \mathrm{p}=.0001)$. Asimismo, se halló que la esta percepción de medidas de reparación se relacionó positivamente con las funciones estatales de $\mathrm{CP}(\mathrm{B}=.22$, $\mathrm{t}(463)=6.90, \mathrm{p}=.0001)$. Por último, la actitud al trabajo de las Comisiones se relacionó positivamente con las funciones estatales de CP (B $=.24, \mathrm{t}(463)=6.41, \mathrm{p}=.0001)$. Luego, los resultados indicaron que el efecto directo de la actitud ante las Comisiones con las funciones estatales de CP siguió siendo significativa $(\mathrm{B}=.15, \mathrm{t}(463)=3.85, \mathrm{p}=$ $.001)$, lo que sugiere una mediación parcial. Estas variables explican el $16 \%$ de la varianza del modelo. 


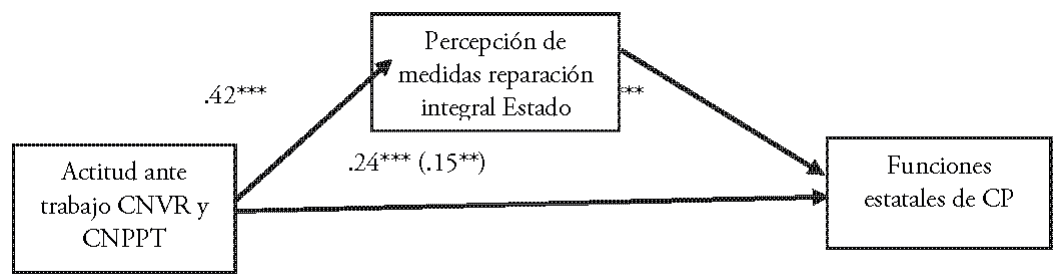

Figura 2. Modelo de mediación parcial entre la percepción de las medidas de reparación integral del Estado, las actitudes del trabajo de la CNVR y CNPPT y las funciones estatales de CP en Chile

Nota CNVR = Comisión Nacional de la Verdad y Reconciliación; CNPPT = Comisión Nacional sobre Prisión Política y Tortura; $\mathrm{CP}=$ Cultura de Paz

\section{Discusión}

Los resultados ponen en evidencia que cuando se activan mecanismos de reparación integral en víctimas que aparecen en los Informes de Comisiones de Verdad, hay mejores condiciones para la construcción de CP. Por el contrario, el incumplimiento de medidas de reparación integral plantea más limitaciones a la hora de generar una construcción de CP y sugiere, asimismo, mayores probabilidades para que se repitan situaciones de violencia.

Respecto a lo encontrado en Chile y Ecuador, la situación parece ser disímil, en el sentido que en Ecuador el Estado ha presentado limitadas respuestas de reparación en las víctimas tras 10 ańos del Informe de la CVE. Por un lado, se pone de manifiesto que el mero conocimiento de las recomendaciones no fomenta la construcción de CP, porque se requiere una tarea más activa y permanente del Estado en pos de reparar a las víctimas. Aunque en Ecuador se ha encontrado una buena evaluación social del impacto positivo del trabajo de la Comisión (ver estudio de Reyes, Grondona-Opazo y Rodríguez, 2015), especialmente en víctimas, hay algunos aspectos que permiten explicar la 
insuficiente respuesta estatal de reparación: la Ley de Víctimas (2013) no cuenta con participación de las víctimas, lo que dificulta un consenso sobre cómo garantizar tales medidas. Además, la dificultad en la judicialización de los responsables y las nulas acciones para generar el cumplimiento de medidas inmateriales, plantean un escenario en el cual ha venido presentándose únicamente una compensación económica, la cual ha sido cuestionada. En suma, el Estado ecuatoriano no ha promovido un cumplimiento integral de medidas de reparación y eso dificulta la construcción de CP.

Por otro lado, los hallazgos presentados en Chile de una percepción positiva al cumplimiento de medidas de reparación integral pueden asociarse al mayor tiempo disponible, casi 30 años, en la cual se han generado otros Informes de Comisiones dada las limitaciones del CNVR. También, se ha encontrado igualmente un impacto positivo del trabajo de las comisiones (véase la investigación de Cárdenas et al., 2013) que, en este estudio, se prolonga para las medidas de reparación integral. En este último punto, las acciones del Estado chileno han abordado la totalidad de las dimensiones de la reparación y cuentan con diversas iniciativas que parecen adelantadas en América Latina. No obstante estos avances, los Informes anuales de derechos humanos (INDH, 2018, 2019) refieren problemas en el cumplimiento de la reparación integral que pueden detallarse en las que siguen: impunidad en responsables de violaciones de derechos humanos, que ha llevado a la liberación de condenados, la nula información y reconocimiento de las fuerzas armadas para informar el paradero de personas desaparecidas y un discurso creciente de desconocimiento de la violencia estatal del periodo informado.

Vistos así, los hallazgos sitúan momentos diferentes en Ecuador y Chile respecto a la activación de medidas de reparación integral, en la cual los Estados no cumplen con las obligaciones establecidas en estándares internacionales (véase "Principios y directrices básicos sobre el derecho de las víctimas de violaciones manifiestas de las normas internacionales de derechos humanos y de violaciones graves del derecho internacional humanitario, Naciones Unidas, 2005) lo que fomenta la impunidad y, por ende, estrecha las condiciones para la construcción de CP. 


\section{Discusión general}

América Latina ha sido una región precursora en abordar las violencias estatales cuya data viene desde la segunda mitad del siglo XX en la aplicación de justicia transicional, que se ha centrado fundamentalmente en promover el reconocimiento de los Estados frente a las violaciones de derechos humanos, el reconocimiento social de las víctimas y la orientación en repararlas integralmente. Sin embargo, a pesar de estos esfuerzos y de considerarse un impacto positivo del trabajo de las Comisiones (Mathías et al., 2020) queda en interrogante el cumplimiento efectivo de las medidas de reparación bajo un enfoque integral. Se reconoce que este mecanismo constituye un aspecto indispensable para la construcción de sociedades equitativas, justas y democráticas que resuelven, en un contexto de justicia, problemas y conflictos que se suscitan y que generan situaciones de vulneración a los derechos humanos.

El presente estudio plantea una relación inédita en las investigaciones en sociedades que han vivido situaciones de violencia con violaciones de derechos humanos, en este caso, ejemplificado para Chile y Ecuador, bajo la siguiente interrogante: ¿De qué modo el cumplimiento de las dimensiones de la reparación integral favorece la construcción de cultura de paz en sociedades posconflictos? Para tal fin, primero se diseña una escala que evalúa fundamentalmente la percepción de $\mathrm{CP}$ en cuatro dimensiones psicosociales: un eje macrosocial asociado a las funciones estatales de CP y los aspectos que afectan la CP y un plano microsocial conformado por las acciones de sociedad civil y las personas y grupos prioritarios en las políticas de CP. Luego, a partir de esta dimensionalidad de CP, se establece la siguiente hipótesis: dado el impacto positivo del trabajo de las Comisiones de Verdad en América Latina, se plantea que las acciones de cumplimiento de las medidas de reparación integral desde los Estados favorecerán las funciones estatales de CP. A partir de esto, se evalúa las relaciones con la actitud hacia el trabajo de las Comisiones y las acciones de reparación integral (en Chile) o el conocimiento de las recomendaciones de reparación integral (en Ecuador). 
Los resultados descriptivos y de los análisis de mediación plantean que hay diferencias en cómo los Estados chileno y ecuatoriano han encarado el cumplimiento de los mecanismos de reparación integral para las víctimas que aparecen en los Informes de las Comisiones de Verdad: en Chile, dado un tiempo más extenso desde la entrega del Informe de la CNVR a lo que se agrega un segundo (CNPPT) considerando víctimas de tortura, se han generado medidas que han contemplado las distintas dimensiones de reparación integral. Esto último es percibido socialmente y posibilita mejores condiciones para la construcción de CP en Chile. En Ecuador, con un espacio de tiempo menor desde la implementación de los mecanismos de justicia transicional, los resultados son más limitados, orientándose única y parcialmente en la dimensión de compensación económica pero con escasos avances en las demás dimensiones, lo que restringe la construcción de CP. Estos resultados sugieren que sean los Estados los que activen medidas en el cumplimiento de la integralidad de la reparación y que, posteriormente, tales sean difundidas y compartidas por la sociedad civil, lo que dará paso al abandono de la coerción como forma de relación entre los Estados y la sociedad civil (Tilly, 2010) y la instauración de reformas normativas y mecanismos institucionales orientados a la paz (Olsen et al., 2016). Estas condiciones serían las más óptimas para la construcción de CP en sociedades posconflictos, en un marco de respeto y garantía de los derechos humanos (ONU A/RES/53/243, 1998; Reyes et al., 2019) que estimula la participación de sociedad civil y de las propias víctimas y se articula una visión heterogénea que propicia la construcción de culturas más pacíficas (de Rivera, 2013), en un contexto de convivencia democrática pacífica (Lira, 2010).

A pesar de resultados aparentemente disímiles, un aspecto imprescindible a considerar se refiere a que el cumplimiento de las medidas de reparación integral no está asociado solo al desarrollo de políticas de atención a las víctimas ni a la promoción de acciones que se orientan a la reparación: el cumplimiento requiere de esfuerzos estatales a través de medidas integradas y articuladas institucionalmente para garantizar la reparación. Esto último resulta aún un aspecto pendiente tanto en 
Chile como en Ecuador, dado que la judicialización y las garantías de no repetición, entre otras medidas, presentan continuas indefiniciones desde los Estados lo que fomenta la tolerancia de discursos que promueven el olvido o la generación de climas emocionales negativos (de Rivera, 2014) y la institucionalización de la violencia (Martín Baró, 1983) que viabiliza la posibilidad de repetir las violencias estatales y generar violaciones de derechos humanos. Las actuales manifestaciones y paro en Ecuador y en Chile han generado respuestas represivas de los Estados, con uso excesivo de la fuerza cometidas por agentes policiales y militares, con diversas violaciones de derechos humanos y un número de personas fallecidas y heridas, que pone en evidencia la vulneración, entre otros, a los derechos de libertad de expresión, a la protesta, a la reunión pacífica y a la participación política de la población (CIDH, 2020). De allí que en un contexto regional con crecientes violaciones a derechos humanos desde los Estados, que acarrean el incumplimiento de mecanismos de reparación integral, la construcción de $\mathrm{CP}$ aparece contradictoria y difícil de imaginar, más que en una $\mathrm{CP}$ inconclusa, compleja y en permanente cambio (Muñoz \& Molina, 2010).

\section{Limitaciones y futuros estudios}

Los estudios presentados aquí presentan limitaciones a considerar: en primer lugar, la muestra de participantes ecuatorianos y chilenos no son representativas, ya que la zona de procedencia de los participantes incluye únicamente a ambas capitales. Sin embargo, para contrarrestar esta limitación, se realiza un muestreo por cuotas por sexo y edad que busca generar más representatividad. En segundo lugar, la conformación de la EDPCP presenta una desigualdad en el número de ítems por dimensión, lo cual ocurre, en particular, en la dimensión de personas y grupos prioritarios de políticas de CP. No obstante lo anterior, se privilegia incorporar a los diferentes grupos que son identificados de atención prioritaria. Una tercera limitación se relaciona de abordar temáticas que no necesariamente cuentan con un grado de conocimiento en los encuestados del trabajo de las Comisiones de Verdad o de las recomendaciones, los cuales, en efecto, presentan una baja 
frecuencia. En este punto, se sigue la estrategia realizada por otros trabajos, de incluir un texto informativo en la encuesta que describe el periodo de violencia en el país, el origen de las Comisiones de Verdad y los hallazgos y recomendaciones.

Futuros estudios requieren reforzar los hallazgos aquí encontrados, principalmente de las diversas formas de construcción de culturas de paz desde sociedad civil. En este sentido, resulta relevante considerar los aportes de movimientos sociales y cómo conciben la CP, ya que sus demandas suelen orientarse más a la defensa y respeto de los derechos humanos (Reyes et al., 2019). Las dimensiones psicosociales obtenidas aquí pueden complementarse con estudios cualitativos que analicen y sistematicen las propuestas de diversos colectivos, en el sentido de cómo se reproduce la violencia, qué estrategias abordan en la resolución de conflictos y cómo entienden la relación con el Estado. Por último, resulta de interés examinar las dinámicas de violencia que se reproducen en distintos ámbitos (e.g., violencia de género, criminalización de defensores, derechos limitados de PPL, participación de fuerzas armadas en paros y movilizaciones sociales, entre otras), poniendo en evidencia la naturalización, pérdida de derechos y dificultad en favorecer consensos sobre la construcción de culturas más pacíficas.

\section{Referencias}

Abad, F., Olea, J., Ponsoda, V., y García, C. (2011). Medición en Ciencias Sociales y de la Salud. Madrid: Síntesis.

Arnoso, M., Cárdenas, M., y Páez, D. (2015). Justicia transicional y predictores del perdón político en Chile. Revista Latinoamericana de Derechos Humanos, 26(1), 77-97.

Batista-Foguet, M., Coenders, G., y Alonso, J. (2004). Análisis factorial confirmatorio. Su utilidad en la validación de cuestionarios. Barcelona, España: Universitat Ramon Llull.

Beristain, C. (2009). Diálogos sobre la reparación. Qué reparar en los casos de violaciones de derechos humanos. Quito: Ministerio de Justicia y Derechos Humanos. 
Beristaín, C., Páez, D., Rimé, B., y Kanyangara, P. (2010). Efectos psicosociales de la participación en rituales de justicia transicional. Revista de Psicología, 28(1), 9-35.

Brahm, E. (2007). Uncoverting the Truth: Examining Truth Commission Success and Impact. Studies Perspectives, 16-35.

Byrne, B. (2006). Structural equation modeling with EQS. Basic concepts, applications, and programming (2nd ed.). Hillsdale, NJ: Lawrence Erlbaum Associates.

Cárdenas, M., Páez, D., Arnoso, M., \& Rimé, B. (2013). El impacto psicosocial de los procesos transicionales en Chile: evaluación de los efectos de las Comisiones Nacional de "Verdad y Reconciliación” y "prisión política y tortura”. Revista de Psicología Social, 28(2), 145-156.

Comisión Interamericana de Derechos Humanos. El Derecho a la Verdad en América. OEA/Ser.L/V/IL.152. Doc 2, 13 de agosto de 2014. Párr. 205.

Dajer, D. (2017). Las garantías de no repetición en el acuerdo final ¿El día después de mañana de la justicia transicional en Colombia? Cuadernos de estrategia, (189), 53-90.

de Rivera, J. (2013). La enseñanza de la cultura de paz como un enfoque de la educación para la paz. En: D. Páez, C.M. Beristain, J.L. González, N. Basabe y J. de Rivera (eds.), Superando la violencia colectiva y construyendo cultura de paz (pp. 569-585). Madrid: Editorial Fundamentos.

de Rivera, J. (2014). Culturas de paz y el clima emocional de las sociedades. En: E. M. Zubieta, J.F. Valencia, and G.I. Delfino (Eds.) Psicología Social y Política. Buenos Aires: EUDEBA.

Feierstein, D. (2009). Terrorismo de Estado y genocidio en América Latina. Buenos Aires: Prometeo libros.

Ferrando, P., y Anguiano-Carrasco, C. (2010). El análisis factorial como técnica de investigación en psicología. Papeles del Psicólogo, 8-33. Ferrando, P. J., \& Lorenzo-Seva, U. (2017). Program FACTOR at 10: Origins, development and future directions. Psicothema, 29(2), 236-240. 
García, S. (2005). La Jurisprudencia de la Corte Interamericana de Derechos Humanos en Materia de Reparaciones. En Corte IDH (Comp.), La Corte Interamericana de Derechos Humanos un Cuarto de Siglo: 1979-2004. Costa Rica, Corte IDH.

George, D., y Mallery, P. (2003). SPSS for Windows step by step: A Simple Guide and Reference 11.0 Update (4ta ed.). Boston: Allyn $\&$ Bacon.

Gibson, J. L. (2004). Overcoming apartheid: Can truth reconcile a divided nation? New York, NY: Russell Sage Foundation.

Hair, J., Anderson, R., Tatham, R., y Black, W. (2004). Análisis multivariante. Madrid, España: Prentice Hall.

Hayes, A. (2017). Introduction to mediation, moderation, and conditional process analysis: A regression-based approach. New York: Guilford Publications.

Informe de la Comisión de la Verdad Ecuador 2010. (2010). Sin verdad no hay justicia. Ecuador: Ediecuatorial.

International Center for Transitional Justice. 2009, Justicia transicional en América Latina: enfrentando los dilemas del presente a partir de los legados del pasado. Disponible en: https://www.ictj.org/sites/ default/files/ICTJ-Global-TransitionalJustice-in-Latin-America-2009-Spanish.pdf

Izquierdo, I., Olea, J., \& Abad, F. J. (2014). Exploratory factor analysis in validation studies: Uses and recommendations. Psicothema, 26(3), 395-400.

Lira, E. (2010). La reparación a las víctimas: Una responsabilidad del Estado. Mensaje, 59(959), 6-11.

Martín Baró, I. (1983). Acción e ideología. Psicología social desde Centroamérica. San Salvador: UCA Editores.

Mathias, A., Páez, D., Espinosa, A., Sandoval, S., Alzugaray, C., Arnoso, M.; Cárdenas, M.; Da Costa, S.; Reyes, C.; Rimé, B y Zubiera, E. (2020). The Association between Truth Commissions Evaluation, Emotional Climate and Institutional Trust: Comparison and Meta-Analysis of Surveys in Six South American Countries. Revista de Psicología Social. (35), 1-30. 
Cumplimiento de medidas de reparación y construcción de cultura de paz / Reyes-Valenzuela et al.

Muñoz, F. (2007). La paz, un anhelo público y político universal. Aportaciones históricas desde el Mediterráneo. Politicas de paz en el mediterráneo, 37-71.

Muñoz, F., y Molina, B. (2010). Una Cultura de Paz compleja y conflictiva. La búsqueda de equilibrios dinámicos. Revista de Paz y Conflictos, 3(3), 44-61.

Nunnally, JC., \& Bernstein, IH. (1994). Psychometric theory (3rd Ed.) New York: McGraw-Hill. Inc.

Olsen, T., Payne, L., y Reiter, A. (2016). Justicia transicional en Equilibrio. Bogotá: Universidad Javeriana.

Organización de las Naciones Unidas (2005). Resolución 60/147. Principios y directrices básicos sobre el derecho de las victimas de violaciones manifiestas de las normas internacionales de derechos humanos y de violaciones graves del derecho internacional humanitario a interponer recursos y obtener reparaciones. ONU.

Organización de las Naciones Unidas (2005). Principios y directrices básicos sobre el derecho de

las victimas de violaciones manifiestas de las normas internacionales de derechos humanos y de violaciones graves del derecho internacional humanitario, ONU.

Organización de las Naciones Unidas, Asamblea General (2017). A/ HRC/WG.6/27/ECU/1. Informe nacional presentado con arreglo al párrafo 5 del anexo de la resolución 16/21 del Consejo de Derechos Humanos. Ecuador.

Organización de las Naciones Unidas, Asamblea General (2017). A/ HRC/WG.6/5/CHL/1. Informe nacional presentado de conformidad con el Párrafo 15 a) anexo a la resolución 5/1 del Consejo de Derechos Humanos. Chile.

Organización de las Naciones Unidas, Comisión de Derechos Humanos (2005). Resolución sobre impunidad, $N^{\circ}$ 2005/81.

Pardo, A., \& San Martón, R. (2010). Análisis de datos en ciencias sociales $y$ de la salud II. Madrid: Editorial Síntesis.

Piper, I. (2005). Memoria y derechos humanos: ¿Prácticas de dominación o resistencia? Santiago de Chile: Universidad ARCIS y CLACSO. 
Reyes, C., Grondona, G., y Rodríguez, M. (2015). Evaluación del Impacto de la Comisión de la Verdad en Ecuador: aportes psicosociales en la actual discusión sobre la reparación a las víctimas. Psicología Política, 15(32), 119-136.

Reyes, C., Bustillos, J., \& Aguirre, A. (2019). El diálogo y articulación entre Estado y sociedad civil en la construcción de Cultura de Paz en Ecuador. Defensa y Justicia, 39, 28-29.

R Core Team. (2019). R: A language and environment for statistical computing. , R Foundation for Statistical Computing. Viena, Austria: R Foundation for Statistical Computing.

Rodrigues, S. (2017). La justicia de transición y las Comisiones de la Verdad en América Latina. Historia Actual Online, 42(1), 157-166.

Rodríguez-Osuna, J. (1991). Métodos de muestreo. En: Cuadernos Metodológicos, núm. 1. Madrid: CIS.

Thompson, B. (2004). Análisis factorial exploratorio y confirmatorio: comprensión de conceptos y aplicaciones. Washington, DC: Asociación Americana de Psicología.

Tilly, C. (2010). Confianza y Gobierno. Buenos Aires: Amorrortu editores.

Valenzuela, L. (2016). Trauma psicosocial: naturaleza, dimensiones y medición. Tesis Doctoral no publicada. Madrid: Universidad Autónoma de Madrid.

van Boven, T., \& Bassiouni, M. (2005). Principios y directrices sobre el derecho de las victimas de violaciones graves a los derechos humanos $y$ al derecho humanitario a obtener reparación.

Winn, P. (2013). La batalla por la memoria histórica en el Cono Sur: conclusiones comparativas.

En: S. J. Stern, P. Winn, F. Lorenz., y A. Marchesi, No hay mañana sin ayer. Batallas por la memoria histórica en el Cono Sur (pp.411-450). Lima: Instituto de Estudios Peruanos.

Recibido: 17 de febrero, 2020

Revisado: 1 de febrero, 2021

Aceptado: 3 de marzo, 2021 


\section{Apéndice 1 \\ Versión de la Escala de dimensiones psicosociales de Cultura de $\mathrm{Paz}$ (EDPCP)}

Item

Dimensión

Usted considera que las políticas de cultura de paz que el Estado ha promovido, han ayudado en el país a:

1. ¿Una reducción de la violencia en el país?

PLANO

2. ¿Una reducción de la desigualdad social?

MACROSOCIAL

3. ¿Un mayor cuidado de la población y de distintos grupos de atención prioritaria? (niños, niñas y adolescentes, mujeres,

Funciones estatales adultos mayores, personas con discapacidad, entre otras)

de Cultura de Paz

4. ¿Una mejor relación entre el Estado y los ciudadanos?

¿Cuánto considera Ud., que afecta en la construcción de una cultura de paz en el país, los siguientes aspectos?

5. Ausencia de judicialización de los casos que aparecen en el Informe de la Comisión de la Verdad

PLANO

MACROSOCIAL

6. Judicialización de casos de corrupción

7. Judicialización de líderes indígenas y/o comunitarios

Factores que afectan

8. Conflictos en partidos políticos

la construcción de

Cultura de Paz

9. Casos de femicidio y violencia contra la mujer

10. Expulsiones de migrantes

¿Cuál de las siguientes acciones y propuestas de paz conoce

PLANO usted en el país?

MICROSOCIAL

12. Acciones de instituciones colaboradoras de Naciones Unidas

13. Actividades desarrolladas en colegios

Iniciativas de

14. Concursos orientados a la paz y no violencia

Cultura de Paz

desde sociedad civil 
¿Cuánto considera usted de las iniciativas y acciones de cultura de paz implementadas por el Estado en el país han favorecido a las siguientes personas o grupos?

15. Víctimas que aparecen en el Informe de la CVE

16. Niños, niñas y adolescentes

PLANO

MICROSOCIAL

17. Mujeres

18. Comunidades indígenas

19. Población LGBTI

20. Población migrante en Ecuador

21. Adultos mayores

22. Personas con discapacidad

23. Personas con enfermedades catastróficas

24. Personas privadas de la libertad

Nota: el ítem 11 fue eliminado.
Personas y grupos prioritarios en las políticas de cultura de paz del Estado 